\title{
Fluorodeoxyglucose positron emission tomography and tumor marker expression in non-small cell lung cancer
}

\author{
Matthew D. Taylor, MD, ${ }^{\text {a }}$ Philip W. Smith, MD, ${ }^{\mathrm{a}}$ William K. Brix, MD, ${ }^{\mathrm{b}}$ Mark R. Wick, MD, ${ }^{\mathrm{b}}$ Nicholas Theodosakis, ${ }^{\mathrm{a}}$ \\ Brian R. Swenson, MD, MS, ${ }^{a}$ Benjamin D. Kozower, MD, ${ }^{a}$ Christine L. Lau, MD, ${ }^{a}$ and David R. Jones, MD ${ }^{a}$
}

\begin{abstract}
Objective: The best current noninvasive surrogate for tumor biology is fluorodeoxyglucose positron emission tomography (FDG-PET). Both FDG-PET maximal standardized uptake values and selected tumor markers have been shown to correlate with stage, nodal disease, and survival in non-small cell lung cancer (NSCLC). However, there are limited data correlating FDG-PET with tumor markers. The purpose of this study was to determine the correlation of tumor marker expression with FDG-PET maximal standardized uptake values in NSCLC.
\end{abstract}

Methods: FDG-PET maximal standardized uptake values were calculated in patients with NSCLC ( $\mathrm{n}=149$ ). No patient had induction chemoradiotherapy. Intraoperative NSCLC tissue was obtained and tissue microarrays were created. Immunohistochemical analysis was performed for 5 known NSCLC tumor markers (glucose transporter 1, p53, cyclin D1, epidermal growth factor receptor, and vascular endothelial growth factor). Each tumor marker was assessed independently by two pathologists using common grading criteria. Subgroup analysis based on histologic characteristics and regional nodal status was performed.

\begin{abstract}
Results: FDG-PET correlated with T classification $(P<.0001)$, N stage $(P=.002)$, and greatest tumor dimension $(P<.0001)$. In addition, increasing maximal standardized uptake values correlated with increased expression of glucose transporter $1(P<.0001)$ and p53 $(P=.04)$ in adenocarcinoma. Epidermal growth factor receptor expression correlated with maximal standardized uptake values without predilection for histologic subtype $(P=.004)$.
\end{abstract}

Conclusion: FDG-PET maximal standardized uptake values correlate with an increased expression of glucose transporter 1 and p53 in lung adenocarcinoma, but not squamous cell cancer. Future studies attempting to correlate FDG-PET with tumor biology will need to consider the effect of different tumor histologic types.

Fluorodeoxyglucose positron emission tomography (FDGPET) has become an important tool in the armamentarium of clinicians for diagnosing and staging of non-small cell lung cancer (NSCLC). FDG-PET maximal standardized uptake values (SUVmax) of primary tumors have been shown to correlate with both stage and nodal disease in NSCLC. ${ }^{1}$ Furthermore, tumor FDG-PET SUVmax have been shown to predict survival in patients with NSCLC. ${ }^{2}$

Although the classic TNM staging system includes widely known prognostic pathologic factors of NSCLC, this system provides no assessment of the biologic or molecular events leading to the development and progression of lung cancer. Molecular pathology has provided considerable information regarding the differential expression of gene products associated with lung carcinogenesis. A recent

\footnotetext{
From the Departments of Surgery ${ }^{\mathrm{a}}$ and Pathology, ${ }^{\mathrm{b}}$ University of Virginia, Charlottesville, Va.

Read at the Thirty-fourth Annual Meeting of The Western Thoracic Surgical Association, Kona, Hawaii, June 25-28, 2008.

Received for publication June 24, 2008; revisions received Sept 18, 2008; accepted for publication Oct 13, 2008

Address for reprints: David R. Jones, MD, Professor of Surgery, University of Virginia, Department of Surgery, Box 800679, Charlottesville, VA 22908-0679 (E-mail: djones@virginia.edu).

J Thorac Cardiovasc Surg 2009; 137:43-8

$0022-5223 / \$ 36.00$

Copyright (c) 2009 by The American Association for Thoracic Surgery

doi:10.1016/j.jtcvs.2008.10.014
}

meta-analysis of immunohistochemical studies investigating tumor marker expression and its correlation to survival in NSCLC found 17 significant tumor markers that have been investigated by eight or more research groups. ${ }^{3}$ With the advent of therapies directed at specific molecular pathways such as epidermal growth factor receptor (EGFR) inhibitors and anti-vascular endothelial growth factor (VEGF) antibodies, analysis of the relationship of molecular markers to diagnostic methods, such as FDG-PET, used to assess treatment response and to provide prognostic information is clearly needed.

Although glucose metabolism is the central factor for increased FDG uptake in tumor cells, very few studies have investigated the correlation of FDG uptake with lung cancer tumor marker expression. The 5 tumor markers selected for evaluation in this study were glucose transporter 1 (GLUT-1), p53, EGFR, VEGF, and cyclin D1. We selected these markers inasmuch as there is ample evidence in the literature suggesting their importance in the development and progression of lung cancer. ${ }^{4-13}$

The purpose of this study is to examine the correlation of selective tumor marker expression to FDG-PET, the bestknown noninvasive surrogate marker of tumor biology. We hypothesize that some or all of these 5 tumor markers will correlate with SUVmax in NSCLC specimens. Subgroup analysis of patients by locoregional nodal status and 


$$
\begin{aligned}
& \text { Abbreviations and Acronyms } \\
& \begin{aligned}
\text { CT } & =\text { computed tomography } \\
\text { EGFR } & =\text { epidermal growth factor receptor } \\
\text { FDG } & =\text { F-deoxyglucose } \\
\text { FDG-PET }= & \text { fluorodeoxyglucose positron } \\
& \text { emission tomography } \\
\text { GLUT-1 } & =\text { glucose transporter } 1 \\
\text { NSCLC } & =\text { non-small cell lung cancer } \\
\text { SUVmax } & =\text { maximal standardized uptake values } \\
\text { VEGF } & =\text { vascular endothelial growth factor }
\end{aligned}
\end{aligned}
$$

histologic characteristics will determine whether expression patterns of the selected tumor markers and FDG-PET are different. Collectively, these studies will offer insight into the correlative relationships between expression of these specific tumor markers and FDG-PET in NSCLC.

\section{PATIENTS AND METHODS \\ Patients}

Paraffin-embedded primary lung tumor samples and patient-matched normal lung tissue in 149 consecutive patients were collected between January 2005 and October 2006. Approval for collection of the patient tissue was obtained from the Institutional Review Board for Health Science Research at the University of Virginia. Individual patient consent was obtained for procurement of tissue for research purposes before patients underwent surgery. Clinicopathologic data were collected from our general thoracic surgery database. Of the 149 patients, 56\% had their FDG-PET scan performed and interpreted at the University of Virginia with a dedicated fusion positron emission tomography/computed tomography (PET/ CT) scanner. The remaining 44\% of patients had their FDG-PET imaging performed at an outside institution with interpretation of the studies independently performed by our nuclear radiologists at the University of Virginia.

\section{Preparation of the Tissue Microarrays}

All tumor slides were reviewed for tumor type and grade by two pathologists. A representative slide and the corresponding block of formalinfixed paraffin-embedded tissue were selected. For each case, three 1-mm cores of tumor and one core of uninvolved epithelium were removed from the original block and embedded in a paraffin block with a specialized manual arraying instrument (model MTA1; Beecher Instruments, Sun Prairie, Wis).

\section{Immunohistochemical Analysis}

A tissue microarray was constructed for immunohistochemical studies that evaluated p53, VEGF, EGFR, cyclin-D1, and GLUT-1. Information for the antibody, antigen retrieval mechanism, and staining pattern are shown in Appendix Table 1. Unstained tissue was used as the negative control. Positive controls are as follows: p53-human lymph node, EGFR-human placenta, GLUT-1-human red blood cells, cyclin D1-human tonsil, and VEGF-colon cancer. Immunohistochemical staining was scored by calculating the percentage of cells staining positive and by assessing the intensity of immunohistochemical staining. Immunohistochemical intensity was scored as $0=$ none, $1=$ weak, $2=$ moderate, and $3=$ intense. The product of each tumor marker's immunohistochemical staining was then calculated by multiplying the percentage of positive cells by the staining intensity. ${ }^{14}$ Product scores ranged from $0 \%$ to $300 \%$.
TABLE 1. Patient demographics

\begin{tabular}{lc}
\hline \multicolumn{1}{c}{ Variable } & Value \\
\hline Patients (N) & 149 \\
Median age (y) & $69($ range, $36-88)$ \\
Sex $(\mathrm{M} / \mathrm{F})$ & $79(53 \%): 70(47 \%)$ \\
Histology & \\
Adenocarcinoma & $68(45.6 \%)$ \\
Squamous & $64(43.0 \%)$ \\
Large cell & $11(7.4 \%)$ \\
Bronchioloalveolar & $6(4.0 \%)$ \\
Pathologic stage & \\
I & $93(62.4 \%)$ \\
II & $35(23.5 \%)$ \\
III & $20(13.4 \%)$ \\
IV & $1(0.7 \%)$ \\
\hline
\end{tabular}

\section{Statistical Analysis}

Statistical analysis was performed with SAS version 9.1 for Windows software (SAS Institute, Inc, Cary, NC). All $P$ values were calculated with the Spearman rank correlation test and the 2-tailed Student $t$ test for continuous variables.

\section{RESULTS}

One hundred forty-nine patients were included in this study. The demographic characteristics of the population are shown in Table 1.

Table 2 illustrates the selected tumor marker correlation coefficients to SUVmax and the respective $P$ values. FDG-SUVmax correlated with greatest tumor dimension, $\mathrm{T}$ classification, and $\mathrm{N}$ classification. There was a positive correlation of increasing SUVmax and increased expression of GLUT- 1 and EGFR in all NSCLC samples. Figure 1 illustrates the expression patterns of these 2 tumor markers in primary lung cancer specimens. Cyclin D1, p53, and VEGF expression did not correlate with SUVmax for our cohort of patients.

NSCLC specimens were next stratified by histologic subtype (adenocarcinoma [ $n=68]$ or squamous $[n=64]$ ) to determine whether the correlation of SUVmax to tumor markers differed by tumor histology. Table 2 shows correlation coefficients of SUVmax with the 5 tumor markers for adenocarcinoma and squamous carcinoma. In adenocarcinoma specimens, GLUT-1 expression strongly correlated with SUVmax, in contrast to squamous histology, in which no significant correlation was found. Also, p53 was found to correlate with SUVmax in adenocarcinoma but not in squamous carcinoma. Furthermore, EGFR expression, found to correlate with SUVmax in the entire cohort, did not reach statistical significance when stratifying by histologic subtype.

We next stratified all patents by FDG-SUVmax and sought to determine whether there was a value that correlated with a significant difference in tumor marker expression. These results are shown in Table 3. Given that SUVmax less than 2.5 in lung tumors are less indicative of 
TABLE 2. Correlation between FDG-SUVmax and tumor marker expression and pathologic stage in all patients with NSCLC (n = 149), lung adenocarcinoma $(n=68)$, and squamous carcinoma $(n=64)$

\begin{tabular}{|c|c|c|c|c|c|c|}
\hline & \multicolumn{2}{|c|}{ All NSCLC specimens } & \multicolumn{2}{|c|}{ Adenocarcinoma } & \multicolumn{2}{|l|}{ Squamous } \\
\hline & Correlation coefficient & $P$ value & Correlation coefficient & $P$ value & Correlation coefficient & $P$ value \\
\hline Greatest tumor dimension & 0.56 & $<.0001$ & 0.36 & .002 & 0.63 & $<.0001$ \\
\hline $\mathrm{T}$ classfication & 0.38 & $<.0001$ & 0.28 & .02 & 0.42 & .0005 \\
\hline $\mathrm{N}$ classification & 0.25 & .002 & 0.26 & .03 & 0.17 & .18 \\
\hline GLUT-1 intensity & 0.36 & $<.0001$ & 0.38 & .001 & 0.10 & .42 \\
\hline GLUT-1 percent & 0.36 & $<.0001$ & 0.47 & $<.0001$ & 0.07 & .56 \\
\hline GLUT-1 product & 0.37 & $<.0001$ & 0.46 & $<.0001$ & 0.07 & .56 \\
\hline p53 intensity & 0.10 & .20 & 0.26 & .03 & -0.14 & .23 \\
\hline p53 percent & 0.11 & .16 & 0.23 & .06 & -0.05 & .66 \\
\hline p53 product & 0.12 & .14 & 0.25 & .04 & -0.09 & .50 \\
\hline EGFR intensity & 0.20 & .01 & 0.12 & .32 & 0.18 & .14 \\
\hline EGFR percent & 0.24 & .003 & 0.17 & .16 & 0.19 & .12 \\
\hline EGFR product & 0.23 & .004 & 0.15 & .21 & 0.19 & .12 \\
\hline Cyclin D1 intensity & -0.06 & .46 & 0.03 & .79 & -0.009 & .94 \\
\hline Cyclin D1 percent & -0.13 & .11 & -0.019 & .87 & -0.07 & .57 \\
\hline Cyclin D1 product & -0.12 & .13 & -0.03 & .79 & -0.05 & .69 \\
\hline VEGF intensity & 0.02 & .79 & 0.21 & .08 & -0.13 & .31 \\
\hline VEGF percent & 0.03 & .66 & 0.21 & .08 & -0.11 & .34 \\
\hline VEGF product & 0.04 & .58 & 0.24 & .06 & -0.04 & .38 \\
\hline
\end{tabular}

GLUT-1, Glucose transporter 1; EGFR, epidermal growth factor receptor; $V E G F$, vascular endothelial growth factor.

malignancy, we used these values as our control group. ${ }^{15}$ We noted a stepwise increase in the percentage of patients with positive regional nodes who had increasing SUVmax. GLUT-1 expression within tumor specimens was significantly higher in patients with SUVmax greater than 10 compared with patients with SUVmax of 2.5 or less. Furthermore, EGFR expression was found to be significantly greater in patients with primary tumor SUVmax greater than 2.5 compared with tumors with SUVmax less than 2.5.

The percentage of NSCLC that had expression of the selected tumor markers and the corresponding mean SUVmax based on regional nodal status is shown in Table 4. SUVmax was significantly greater for tumors with regional node-positive disease ( $6.4 \pm 0.5$ vs $8.5 \pm 0.6 ; P=.005)$. GLUT- 1 expression was found in a significantly greater proportion of tumors with associated regional nodal disease as compared with GLUT-1 expression in tumors without nodal disease. In the proportion of tumors demonstrating expression of GLUT-1, p53, and cyclin D1, SUVmax was significantly greater in the tumors with associated regional nodal disease than in those with node-negative disease.

\section{DISCUSSION}

This study investigates the correlation of 5 tumor markers in lung cancer with the best noninvasive method of assessing tumor biology, FDG-PET. Previous studies have demonstrated the correlation of FDG-PET SUVmax with survival in patients with lung cancer. ${ }^{2,16}$

GLUT-1 overexpression, the human erythrocyte glucose transporter, has been correlated with a poor prognosis in pa- tients with lung cancer. ${ }^{17}$ Increased glucose uptake in tumor cells is thought to be regulated by glucose transporter activity. ${ }^{5}$ In our study, SUVmax was found to positively correlate with the expression of GLUT-1 in NSCLC and specifically adenocarcinoma. These results support similar findings reported in other studies. ${ }^{18,19}$ In addition, we demonstrated that the percentage of positive GLUT-1 tumor cells was significant higher between those patients with primary tumor SUVmax greater than 10 when compared with those with values less than 2.5. Stratifying this group of patients with lung cancer by regional nodal status, we found that the number of lung cancers expressing GLUT-1 was significantly higher in specimens with concurrent regional nodal disease. Furthermore, SUVmax of these GLUT-1 positive tumors with regional nodal disease was significantly greater than SUVmax for node-negative tumors. One previous study demonstrated a correlation of GLUT-1 expression in primary lung tumor with metastatic lymph nodes; however, no comparison was made between GLUT-1 expression of the tumor and its nodal status. ${ }^{20}$

Alterations of tumor suppressor genes such as $\mathrm{p} 53$ by mutation or overexpression are frequently found in lung carcinoma, with many studies, although not all, suggesting that is associated with a poor prognosis. ${ }^{6,11,13}$ Our analysis of p53 expression in NSCLC, demonstrated a significant correlation of p53 overexpression with SUVmax in lung adenocarcinoma. This is the first larger cohort study demonstrating a correlation between $\mathrm{p} 53$ expression and SUVmax in lung cancer. SUVmax of p53 immunopositive tumors with regional nodal disease were significantly greater than SUVmax for node-negative tumors. We did not find 

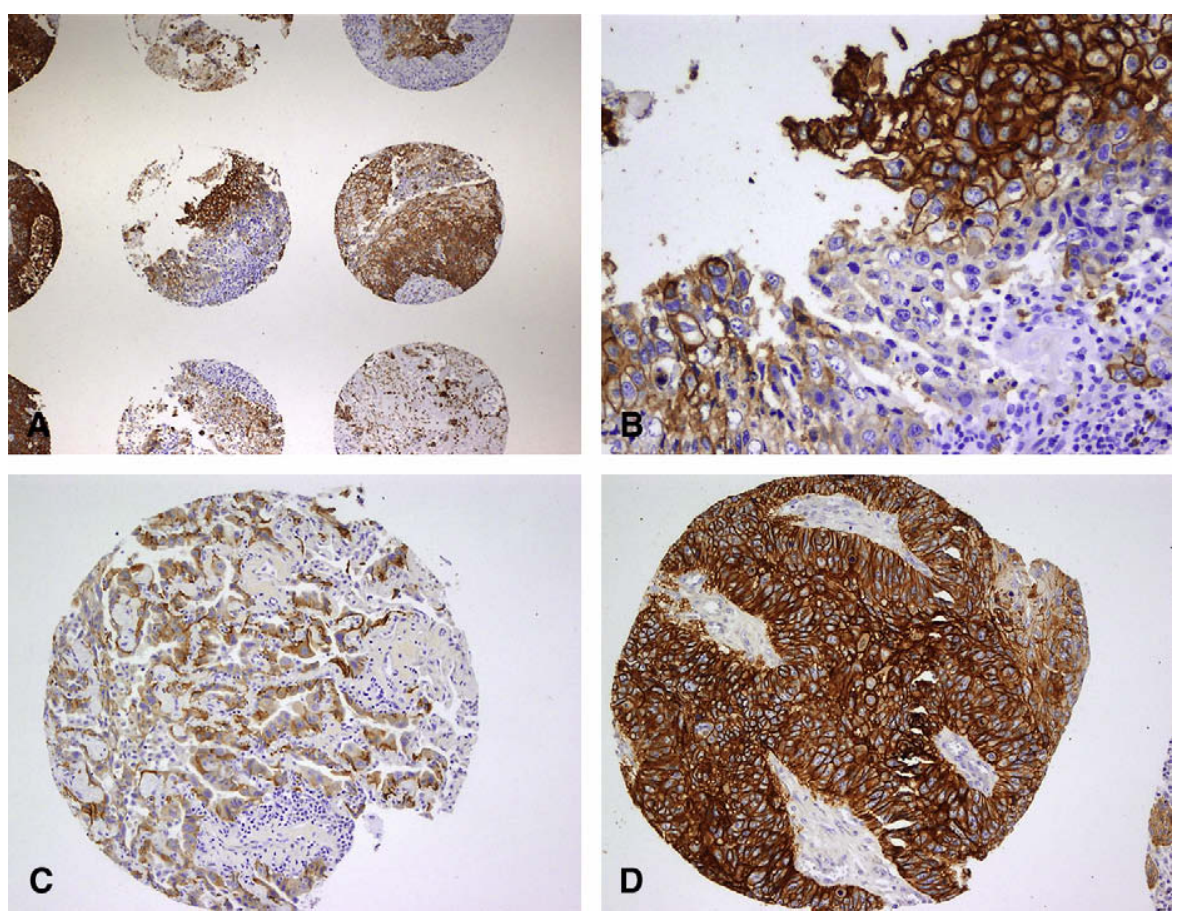

FIGURE 1. NSCLC tissue microarray. A, Representative $2 \times$ magnification tissue microarray of GLUT-1 expression in NSCLC demonstrates differential expression patterns between patient tumor samples. B, Representative $20 \times$ magnification illustrating diffuse GLUT- 1 expression in NSCLC. C, Representative $10 \times$ magnification tissue microarray of EGFR expression in lung adenocarcinoma. D, Representative $10 \times$ magnification tissue microarray of EGFR expression in lung squamous carcinoma. NSCLC, Non-small cell lung cancer; GLUT-1, glucose transporter 1; EGFR, epidermal growth factor receptor.

a significant increase in the number of NSCLCs expressing p53 on the basis of regional nodal status. Aberrant expression of p53 in NSCLC has been associated with an increased likelihood of mediastinal nodal metastasis. ${ }^{21}$ The discrepancy between our results and that study may be related to the 3fold higher representation of pathologic stage III and IV disease in that study.

EGFR expression appears to have moderate prognostic value in NSCLC. ${ }^{9}$ Specific EGFR-activating mutations within lung tumors have been associated with a dramatically improved response to EGFR inhibitors in early-phase clinical trials. ${ }^{22}$ EGFR expression was found to correlate to SUVmax in the entire cohort; however, statistical significance was not obtained when the specimens were examined by histologic subtype. This discrepancy is likely a result of the reduction in sample size associated with stratification. Also, this study illustrated that EGFR expression was significantly greater in NSCLC with SUVmax greater than 2.5. These results support the clinical utility of FDG-PET as a means of assessing response to EGFR inhibitor therapy, as suggested by other groups. ${ }^{22}$ We did not find a significant difference in EGFR expression on the basis of tumor nodal status. Additionally, SUVmax of EGFR-positive tumors with regional nodal disease were not found to be greater for similar tumors without nodal disease. Fontanini and associates $^{23}$ found a correlation of EGFR expression in pri- mary squamous cell lung cancers with the presence of node-positive disease. However, this correlation was not found in patients with lung adenocarcinoma. Our study had an equal number of patients with squamous and adenocarcinoma histologic features, and there were fewer patient samples examined, both of which may account for the discrepancy between our study and that one.

Cyclin D1, an important cell cycle regulator, is translocated to the cell nucleus when exposed to tobacco carcinogens, and this nuclear accumulation induces uncontrolled cell proliferation. ${ }^{7}$ Cyclin D1 overexpression has been demonstrated in NSCLC compared with normal lung tissue. ${ }^{4}$ SUVmax did not correlate with the expression of cyclin D1. Other studies have reported similar findings. ${ }^{24} \mathrm{We}$

TABLE 3. FDG-PET SUVmax and tumor marker expression

\begin{tabular}{lcccc}
\hline & & \multicolumn{2}{c}{$\begin{array}{c}\text { Immunohistochemistry } \\
\text { percent scores (mean } \pm \text { SEM) }\end{array}$} \\
\cline { 3 - 5 } SUVmax & No. positive (\%) & GLUT-1 (\%) & EGFR (\%) \\
\hline $0-2.5$ & 25 & $1 / 25(4 \%)$ & $28 \pm 6$ reference & $10 \pm 4$ reference \\
$2.5-5.0$ & 37 & $10 / 37(27 \%)$ & $39 \pm 6 P=.25$ & $27 \pm 6 P=.02 *$ \\
$5-10$ & 53 & $20 / 53(38 \%)$ & $44 \pm 5 P=.06$ & $34 \pm 5 P=.0003 *$ \\
10 or greater & 34 & $14 / 34(41 \%)$ & $65 \pm 6 P=.0001 *$ & $37 \pm 7 P=.002 *$ \\
\hline
\end{tabular}


TABLE 4. Tumor marker expression and SUVmax by nodal status

\begin{tabular}{|c|c|c|c|c|c|c|}
\hline \multirow[b]{2}{*}{ Tumor marker } & \multicolumn{2}{|c|}{ Node-negative $(n=104)$} & \multicolumn{2}{|c|}{ Node-positive $(n=45)$} & \multicolumn{2}{|c|}{$P$ value } \\
\hline & Percent positive $(\%)$ & Mean SUVmax* & Percent positive $(\%)$ & Mean SUVmax* & Percent positive (\%) & Mean SUVmax \\
\hline GLUT-1 & 73 & $7.0 \pm 0.6$ & 87 & $8.9 \pm 0.6$ & .03 & .02 \\
\hline p53 & 75 & $6.4 \pm 0.5$ & 78 & $8.3 \pm 0.7$ & .16 & .03 \\
\hline Cyclin D1 & 81 & $6.3 \pm 0.5$ & 78 & $8.4 \pm 0.6$ & .16 & .01 \\
\hline VEGF & 52 & $6.4 \pm 0.6$ & 53 & $8.2 \pm 0.9$ & .14 & .08 \\
\hline EGFR & 52 & $7.6 \pm 1.0$ & 49 & $8.8 \pm 1.9$ & .43 & .28 \\
\hline
\end{tabular}

SUVmax, Maximal standardized uptake values; GLUT-1, glucose transporter 1; VEGF, vascular endothelial growth factor; EGFR, epidermal growth factor receptor. $*$ Mean \pm standard error of the mean.

also did not find a significant difference in cyclin D1 expression based on tumor nodal status. However, SUVmax in cyclin D1-positive tumors with regional node-positive disease was significantly greater than in tumors without nodal disease. Cyclin D1 overexpression in NSCLC has been associated with lymph node metastasis. ${ }^{25}$ Closer inspection of these data supports that cyclin D1 overexpression and nodal disease were significantly stronger for stage III $(P=.001)$ versus stage I or II $(P=.048)$ disease. In this study $41 \%$ of patients had stage III disease, whereas in our study only $13 \%$ had stage III disease.

VEGF plays a critical role in tumor angiogenesis and metastasis in NSCLC. Studies have demonstrated overexpression of VEGF in NSCLC. ${ }^{8,10}$ A recent review of angiogenesis in NSCLC uncovered 16 studies highlighting the prognostic significance of VEGF overexpression in lung cancer. ${ }^{26}$ VEGF expression did not correlate with SUVmax in our population. Our study is the first to define the relationship of VEGF expression to FDG-PET in lung cancer. Stratification by regional nodal disease found no significant difference in VEGF expression based on tumor nodal status. However, an insignificant trend favoring increased SUVmax of VEGF-positive tumors with nodal disease compared with similar tumors with node-negative disease was noted. Previous studies investigating the correlation of VEGF expression in NSCLC and the presence of nodal disease have had discordant conclusions. ${ }^{27-30}$ Therefore, in this complex milieu of growth factors, VEGF expression alone in lung tumors is unlikely to predict the presence of lymph node metastasis.

Limitations of this study include the limited sample size used. A larger sample size may elucidate further relationships between FDG-PET and the investigated tumor markers. Second, use of tissue microarrays in which small tissue cylinders are studied may not in fact adequately represent the entire specimen owing to tissue heterogeneity. Third, despite our efforts to use a well-established protocol, inherent drawbacks of immunohistochemical analysis include interlaboratory differences in antigen retrieval, staining protocols, and antibodies used. A fourth limitation is that PET/CT imaging was performed on multiple scanners, which could influence the interpretation of SUVmax. We attempted to address this by having our nuclear radiologists interpret the images at our institution.

Future study directions will evaluate expression of these tumor markers at a transcriptional level using a more sensitive method, quantitative real-time polymerase chain reaction. This investigation will use cryopreserved lung tumors, thereby circumventing the potential pitfall of using paraffin-embedded tissue for the assessment of transcriptional regulation.

In conclusion, GLUT-1 and EGFR expression in primary NSCLC correlates with FDG-SUVmax. The correlation between SUVmax and GLUT-1 expression is significantly stronger in lung adenocarcinoma. Additionally, p53 expression significantly correlated with SUVmax in lung adenocarcinoma. GLUT-1 expression in NSCLC is significantly greater in patients with regional mediastinal nodal disease. In tumors expressing GLUT-1, p53, and cyclin D1, SUVmax was significantly greater in patients with regional nodal disease. Finally, the correlation of EGFR expression with FDG-PET in lung cancer suggests potential clinical utility in monitoring response to EGFR inhibitor therapy. In contrast, a lack of correlation between FDG-PET and VEGF expression points to limited utility of FDG-PET to assess clinical response of lung adenocarcinoma to anti-VEGF therapies.

\section{References}

1. Vansteenkiste JF, Stroobants SG, Dupont PJ, De Leyn PR, Verbeken EK, Deneffe GJ, et al. Prognostic importance of the standardized uptake value on (18)F-fluoro-2-deoxy-glucose-positron emission tomography scan in nonsmall-cell lung cancer: An analysis of 125 cases. Leuven Lung Cancer Group. J Clin Oncol. 1999; 17:3201-6.

2. Cerfolio RJ, Bryant AS, Ohja B, Bartolucci AA. The maximum standardized uptake values on positron emission tomography of a non-small cell lung cancer predict stage, recurrence, and survival. J Thorac Cardiovasc Surg. 2005;130:151-9.

3. Zhu CQ, Shih W, Ling CH, Tsao MS. Immunohistochemical markers of prognosis in non-small cell lung cancer: a review and proposal for a multiphase approach to marker evaluation. J Clin Pathol. 2006;59:790-800.

4. Betticher DC, Heighway J, Hasleton PS, Altermatt HJ, Ryder WD, Cerny T, et al. Prognostic significance of CCND1 (cyclin D1) overexpression in primary resected non-small-cell lung cancer. Br J Cancer. 1996;73:294-300.

5. Flier JS, Mueckler MM, Usher P, Lodish HF. Elevated levels of glucose transport and transporter messenger RNA are induced by ras or src oncogenes. Science. 1987;235:1492-5.

6. Fujino M, Dosaka-Akita H, Harada M, Hiroumi H, Kinoshita I, Akie K, et al. Prognostic significance of p53 and ras p21 expression in nonsmall cell lung cancer. Cancer. 1995;76:2457-63. 
7. Gautschi O, Ratschiller D, Gugger M, Betticher DC, Heighway J. Cyclin D1 in non-small cell lung cancer: a key driver of malignant transformation. Lung Cancer. 2007;55:1-14.

8. Kajita T, Ohta Y, Kimura K, Tamura M, Tanaka Y, Tsunezuka Y, et al. The expression of vascular endothelial growth factor $\mathrm{C}$ and its receptors in non-small cell lung cancer. Br J Cancer. 2001;85:255-60.

9. Nicholson RI, Gee JM, Harper ME. EGFR and cancer prognosis. Eur J Cancer. 2001;37(Suppl. 4):S9-15.

10. Niklinska W, Burzykowski T, Chyczewski L, Niklinski J. Expression of vascular endothelial growth factor (VEGF) in non-small cell lung cancer (NSCLC): association with p53 gene mutation and prognosis. Lung Cancer. 2001;34(Suppl. 2):S59-64.

11. Quinlan DC, Davidson AG, Summers CL, Warden HE, Doshi HM. Accumulation of 553 protein correlates with a poor prognosis in human lung cancer. Cancer Res. 1992;52:4828-31.

12. Sunaga N, Oriuchi N, Kaira K, Yanagitani N, Tomizawa Y, Hisada T, et al. Usefulness of FDG-PET for early prediction of the response to gefitinib in non-small cell lung cancer. Lung Cancer. 2008;59:203-10.

13. Takahashi T, Nau MM, Chiba I, Birrer MJ, Rosenberg RK, Vinocour M, et al. p53: a frequent target for genetic abnormalities in lung cancer. Science. 1989; 246:491-4

14. Lagendijk JH, Mullink H, van Diest PJ, Meijer GA, Meijer CJ. Immunohistochemical differentiation between primary adenocarcinomas of the ovary and ovarian metastases of colonic and breast origin. Comparison between a statistical and an intuitive approach. J Clin Pathol. 1999;52:283-90.

15. Al-Sugair A, Coleman RE. Applications of PET in lung cancer. Semin Nucl Med. 1998;28:303-19.

16. Ahuja V, Coleman RE, Herndon J, Patz EF Jr. The prognostic significance of fluorodeoxyglucose positron emission tomography imaging for patients with nonsmall cell lung carcinoma. Cancer. 1998;83:918-24.

17. Younes M, Brown RW, Stephenson M, Gondo M, Cagle PT. Overexpression of Glut 1 and Glut3 in stage I nonsmall cell lung carcinoma is associated with poor survival. Cancer. 1997;80:1046-51.

18. de Geus-Oei LF, van Krieken JH, Aliredjo RP, Krabbe PF, Frielink C, Verhagen AF, et al. Biological correlates of FDG uptake in non-small cell lung cancer. Lung Cancer. 2007;55:79-87.

19. van Baardwijk A, Dooms C, van Suylen RJ, Verbeken E, Hochstenbag M, Dehing-Oberije $\mathrm{C}$, et al. The maximum uptake of (18)F-deoxyglucose on positron emission tomography scan correlates with survival, hypoxia inducible factor-1alpha and GLUT-1 in non-small cell lung cancer. Eur J Cancer. 2007;43:1392-8.
20. Nguyen XC, So Y, Chung JH, Lee WW, Park SY, Kim SE. High correlations between primary tumours and loco-regional metastatic lymph nodes in nonsmall-cell lung cancer with respect to glucose transporter type 1-mediated 2-deoxy-2-F18-fluoro-D-glucose uptake. Eur J Cancer. 2008;44:692-8.

21. Tanaka F, Yanagihara K, Otake Y, Li M, Miyahara R, Wada H, et al. Biological features and preoperative evaluation of mediastinal nodal status in non-small cell lung cancer. Ann Thorac Surg. 2000;70:1832-8.

22. Sunaga N, Tomizawa Y, Yanagitani N, Iijima H, Kaira K, Shimizu K, et al. Phase II prospective study of the efficacy of gefitinib for the treatment of stage III/IV non-small cell lung cancer with EGFR mutations, irrespective of previous chemotherapy. Lung Cancer. 2007;56:383-9.

23. Fontanini G, Vignati S, Bigini D, Mussi A, Lucchi H, Angeletti CA, et al. Epidermal growth factor receptor (EGFr) expression in non-small cell lung carcinomas correlates with metastatic involvement of hilar and mediastinal lymph nodes in the squamous subtype. Eur J Cancer. 1995;31A:178-83.

24. Watanabe K, Nomori H, Ohtsuka T, Naruke T, Ebihara A, Orikasa H, et al. [F18]Fluorodeoxyglucose positron emission tomography can predict pathological tumor stage and proliferative activity determined by Ki-67 in clinical stage IA lung adenocarcinomas. Jpn J Clin Oncol. 2006;36:403-9.

25. Keum JS, Kong G, Yang SC, Shin DH, Park SS, Lee JH, et al. Cyclin D1 overexpression is an indicator of poor prognosis in resectable non-small cell lung cancer. Br J Cancer. 1999;81:127-32.

26. Bremnes RM, Camps C, Sirera R. Angiogenesis in non-small cell lung cancer: the prognostic impact of neoangiogenesis and the cytokines VEGF and bFGF in tumours and blood. Lung Cancer. 2006;51:143-58.

27. Yuan A, Yu CJ, Chen WJ, Lin FY, Kuo SH, Luh KT, et al. Correlation of total VEGF mRNA and protein expression with histologic type, tumor angiogenesis, patient survival and timing of relapse in non-small-cell lung cancer. Int J Cancer. 2000;89:475-83.

28. Arinaga M, Noguchi T, Takeno S, Chujo M, Miura T, Uchida Y. Clinical significance of vascular endothelial growth factor $\mathrm{C}$ and vascular endothelial growth factor receptor 3 in patients with nonsmall cell lung carcinoma. Cancer. 2003; 97:457-64.

29. Ogawa E, Takenaka K, Yanagihara K, Kurozumi M, Manabe T, Wada H, et al. Clinical significance of VEGF-C status in tumour cells and stromal macrophages in non-small cell lung cancer patients. Br J Cancer. 2004;91:498-503.

30. Takizawa H, Kondo K, Fujino H, Kenzaki K, Miyoshi T, Sakiyama S, et al. The balance of VEGF-C and VEGFR-3 mRNA is a predictor of lymph node metastasis in non-small cell lung cancer. Br J Cancer. 2006;95:75-9.

Appendix TABLE 1. Antibodies for immunohistochemical analysis

\begin{tabular}{lcclllll}
\hline Antibody & Company & Catalog No. & \multicolumn{1}{c}{ Type } & \multicolumn{1}{c}{ Clone } & Dilution & Antigen retrieval & Positive staining pattern \\
\hline VEGF & DAKO & M7273 & Monoclonal mouse & VG1 & $1 / 6400$ & Citrate buffer & Cytoplasmic \\
EGFR & DAKO & M3563 & Monoclonal mouse & H11 & $1 / 400$ & Citrate buffer & Cytoplasmic \\
p53 & DAKO & M7001 & Monoclonal mouse & DO-7 & $1 / 50$ & Citrate buffer & Nuclear \\
GLUT-1 & DAKO & A3536 & Polyclonal rabbit & Not available & $1 / 300$ & Citrate buffer & Cytoplasmic membrane \\
Cyclin D1 & DAKO & M7155 & Monoclonal mouse & DCS-6 & $1 / 50$ & Citrate buffer & Nuclear \\
\hline
\end{tabular}

$V E G F$, Vascular endothelial growth factor; $E G F R$, epidermal growth factor receptor; GLUT-1, glucose transporter 1. 